\title{
A Case Report on Acquired Pure Red Cell Aplasia in a Patient of Rheumatoid Arthritis
}

\author{
Authors \\ Kumar Rajesh ${ }^{1}$, Pradhan $\mathbf{R N}^{2}$, Bhattiprolu $\mathbf{R K}^{3}$ \\ ${ }^{1,2,3}$ Department of Medicine, B.G.H. DNB Hospital, Bokaro India \\ Email: ${ }^{1}$ drrajeshdr@yahoo.co.in, ${ }^{2}$ rabindranath.dr@gmail.com, ${ }^{3}$ rahikiran73@gmail.com \\ Corresponding Author \\ Kumar Rajesh \\ Department of Medicine, B.G.H. DNB Hospital, Bokaro India
}

\begin{abstract}
Pure red cell aplasia (PRCA) is a clinical syndrome defined by the absence of mature erythroid precursors in another wise normocellular bone marrow. PRCA is considered to be an extra articular manifestation of rheumatoid arthritis and is very rare cause of anemia in Rheumatoid Arthritis with only very few cases reported till now. Herein we report a case of 52 year old female with long standing untreated seropositive arthritis who presented to us with severe symptomatic anemia. After proper work up including bone marrow examination, her findings were consistent with pure red cell aplasia.

Key words: Anemia, Pure Red Cell Aplasia, Rheumatoid arthritis
\end{abstract}

Abbreviation used: CLL - chronic lymphocytic anemia, DMARDs - disease modifying anti-rheumatic drugs, PND - paroxysmal nocturnal dyspnea,ESR-erythrocyte sedimentation rate, CRP- C Reactive protein, NSAID- non steroidal anti-inflammtory drugs, RPI- Reticulocyte production index

\section{INTRODUCTION}

Pure red cell aplasia (PRCA) is a heterogenous disorder characterized by selective reduction in marrow erythroid elements. Patients have severe anemia, low corrected reticulocyte count, normal platelet and granulocyte counts ${ }^{1,2}$. PRCA may be classified into hereditary or acquired. Acquired PRCA is an uncommon cause of anemia that occurs principally in older adults. The nosologic origins of acquired PRCA are obscure. PRCA is associated with auto immune diseases ${ }^{2}$ (rheumatoid arthritis, SLE, adult onset still disease) thymoma, lympho proliferative disorder (CLL, Hodgkins lymphoma), drugs ${ }^{3}$ (phenytoin, isoniazid etc.), Darbepoetin ${ }^{4}$, infections ${ }^{7}$ (parvo virus B19, Epstein-Bar rvirus ).

The bone marrow findings for diagnosis of PRCA by Dessypris ${ }^{5}$ includes; reticulocyte count below $1 \%$, no major changes in white cell count or platelet count, normal cellularity of bone marrow, less than $1 \%$ erythroblasts (occasionally upto $5 \%$ proerythroblasts or basophilic erythroblasts), normal myeloid cells and megakaryocytes.

The exact pathophysiology of anemia in rheumatoid arthritis is multifactorial mainly anemia of chronic disease and nutritional anemia. Other causes include bone marrow suppression, gastrointestinal bleed and rarely $\mathrm{PRCA}^{2}$. The 
pathogenesis of PRCA is by both antibody and cellular mechanisms of inhibition of erythropoiesis.

\section{CASE HISTORY}

A 52yearold female was admitted with symptoms of severe anemia. It started 18 months back with generalized weakness, fatigueness, lassitude, reduced appetite followed by progressive increase in breathlessness on exertion since past six months. There was no history of PND, orthopnoea, bleeding from any site. She had chronic polyarthritis with deformities (Fig 1) since last ten years. She used to take NSAID'S occasionally for pain but was not on any DMARDs or any alternative medications. She had been treated outside with history of four units of blood transfusion in past six months and was subsequently referred to us. Relevant findings included, marked pallor with pitting pedal edema, joint deformities with DAS 28 score of 5.08(moderate disease activity). On investigation, $\mathrm{Hb} \%$ was decreased $(3.30 \mathrm{gm} \%)$ with normal total leucocyte normal platelets, decreased hematocrit, reticulocyte count $(0.90 \%)$ with RPI of 0.1008 . $\mathrm{MCV}, \mathrm{MCH}, \mathrm{MCHC}$ were normal with decreased RBC count, increased ESR and CRP (18 mg/l). normocytic, normochromic anemia with adequate platelets. Serum ferritin was increased (602.2microgm/l) with normal TIBC and serum iron. RA factor and anti-CCP was positive, ANA and Anti ds DNA was negative .Renal and liver function test, lipid profile, LDH and TSH levels were normal. Stool for occult blood was negative. Interestingly on bone marrow aspiration, there was moderately hypercellular marrow with marked erythroid hypoplasia, adequate iron stores (Fig 2). The findings were consistent of PRCA. CT-thorax ruled out thymoma, USG-abdomen and upper GI endoscopy were normal, serological workup for infections like Parvo virus B19, Epstein Barr virus, viral hepatitis, HIV were negative.

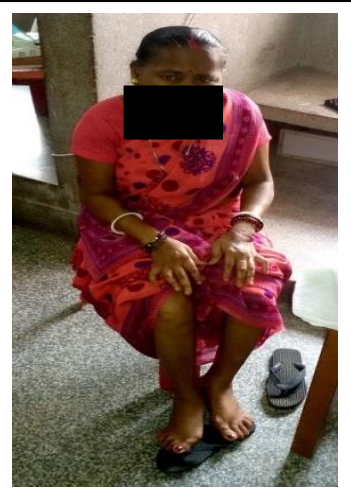

Figure 1: Showing the patient of Rheumatoid arthritis with hand deformities diagnosed with PRCA

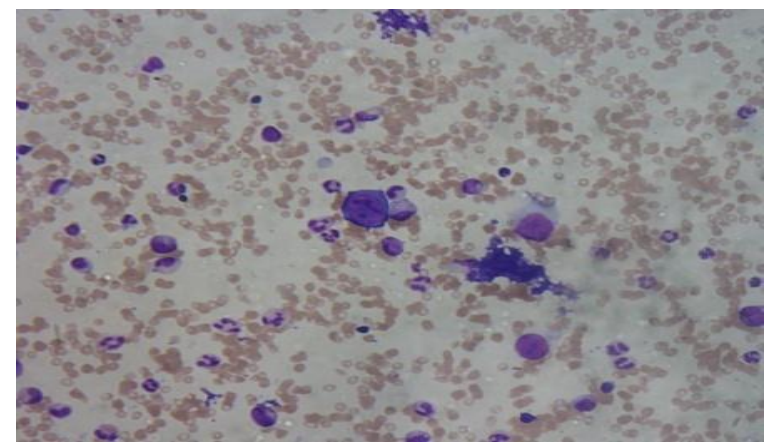

Figure 2: Bone marrow aspiration slide showing moderately hypercellular marrow with marked erythroid hypoplasia, eosinophilia, moderate lymphocytosis and adequate iron store, which are consistent features of PRCA

\section{CASE DISCUSSION}

To date, there appears to be no consensus on the diagnostic criteria of PRCA due to RA. Diagnosis is established by exclusion of the most common causes of acquired PRCA and classic findings on bone marrow examination (Dessypriss ${ }^{5}$ ). Majority of patients with RA have mild anemia (normocytic, normochromic) that correlates with the activity of the disease ${ }^{2}$. Our patient presented with severe anemia, low corrected reticulocyte count, normal platelet and granulocyte counts for which she required multiple blood transfusions without history of acute or chronic blood loss which is unusual with disease like RA.

Bone marrow examination revealed decreased erythroblasts with normal myeloid and megakaryocytic cells. These findings were consistent with features of PRCA ${ }^{1,2}$. She had no 
history of intake of drugs ${ }^{3,5}$ including DMARD's. Other causes of acquired PRCA such as thymoma, lymph proliferative disease ${ }^{2}$, infection, were ruled out by imaging studies and serological tests respectively. Thus diagnosis of acquired PRCA on background of long standing RA was kept.

Prednisolone was given ( $1 \mathrm{mg} / \mathrm{kg}$ body wt) initially for one month but seeing no significant response, was subsequently switched to cyclosporine therapy wherein patient responded. Bone marrow repeated after 2 two months after treatment showed significant rise of erythroid precursors in different stages of differentiation. Other treatment modalities available are Antithymocyte globulin, plasmapheresis, rituximab, anti CD-52 alemtuzumab, autologous bone marrow transplant.

\section{CONCLUSION}

Acquired PRCA is a rare cause of anemia in rheumatoid arthritis and should be considered when there is severe anemia with characteristic bone marrow appearance.

\section{BIBLIOGRAPHY}

1. Charles RJ, Sabo KM, Kidd PG, Abkowitz JL. The Pathophysiology of Pure Red Cell Aplasia: Implications for Therapy. Blood. 1996 June 1;87:4831-38.

2. Bandyopadhyay SK, Bandyopodhyay R, Dutta A. Acquired pure red cell aplasia in a patient of Rheumatoid arthritis and autoimmune hypothyroidism. J Indian Rheumatol Assoc 2003;11: 87-8

3. Malipatil RA, Patil SY. Drug Induced Chronic Acquired Pure Red cell Aplasia in Adolescent boy. NJIRM 2011; Vol. 2(3). July- September ;2(3):98-100.

4. Howman R, Kulkarni H. Antibodymediated acquired pure red cell aplasia (PRCA) after treatment with darbepoetin. Nephrol Dial Transplant . 2007; 22: 146264.

5. Vlachaki E, Diamantidis MD, Klonizakis P, Haralambidou-Vranitsa S, Elizabeth
Ioannidou-Papagiannaki, Klonizakis L. Pure Red Cell Aplasia and Lymph proliferative Disorders: An Infrequent Association. The Scientific World Journal. 2012; 2012:1-7. doi:10.1100/2012/475313. 